\title{
Before The Pandemic Ends: Making Sure This Never Happens Again
}

Daniel R. Brooks ${ }^{1}$, Department of Ecology and Evolutionary Biology, University of Toronto (emeritus); Harold W. Manter Laboratory of Parasitology, University of Nebraska-Lincoln, NE 68588-0514; Institute for Evolution, Centre for Ecological Research, Karolina ut 29, Budapest, Hungary, H-1113 corresponding author: dnlbrooks@gmail.com.

Eric P. Hoberg ${ }^{2}$, Pathobiological Sciences, School of Veterinary Medicine, University of Wisconsin-Madison; Museum of Southwestern Biology, Department of Biology, University of New Mexico

Walter A. Boeger ${ }^{3}$, Biological Interactions, Universidade Federal do Paraná, Cx Postal 19073, Curitiba, Brazil

Scott L. Gardner ${ }^{4}$, Harold W. Manter Laboratory of Parasitology, University of Nebraska-Lincoln, NE 68588-0514

Sabrina B.L. Araujo ${ }^{5}$, Biological Interactions, Departamento de Física, Universidade Federal do Paraná, Cx Postal 19073, Curitiba, Brazil

Katalin Bajer ${ }^{6}$, Department of Systematic Zoology and Ecology, Eötvös Loránd University, Pázmány Péter ave. 1/C, Budapest, Hungary, $\mathrm{H}-1117$

Sebastian Botero-Cañola7, Harold W. Manter Laboratory of Parasitology, University of Nebraska-Lincoln, NE 68588-0514 Brian Byrd ${ }^{8}$, Mosquito \& Vector-borne Infectious Disease Laboratory, Western Carolina University, Cullowhee, NC 28723 USA

Gábor Földvári ${ }^{9}$, Institute of Evolution, Centre for Ecological Research, Karolina ut 29, Budapest, Hungary, H-1113 Joseph A. Cook ${ }^{10}$, Museum of Southwestern Biology and Biology Department, University of New Mexico, Albuquerque, New Mexico, 87131-0001 
Jonathan Dunnum ${ }^{11}$, Museum of Southwestern Biology and Biology Department, University of New Mexico, Albuquerque, New Mexico, 87131-0001

Altangerel Tsogtsaikhan Dursahinhan ${ }^{12}$, Harold W. Manter Laboratory of Parasitology, University of Nebraska-Lincoln, NE 68588-0514

László Zsolt Garamszegi'13, Institute of Ecology and Botany, Centre for Ecological Research, H-2163 Vácrátót, Alkotmány u. 2-4., Hungary

Dávid Herczeg ${ }^{14}$, Lendület Evolutionary Ecology Research Group, Plant Protection Institute, Centre for Agricultural Research, Budapest, Hungary

Ferenc Jakab ${ }^{15}$, Virological Research Group, Szentágothai Research Centre, University of Pécs and Institute of Biology, Faculty of Sciences, University of Pécs

Alicia Juarrero ${ }^{16}$, CEO, VectorAnalytica, Washington DC

Ferenc Jakab17, Virological Research Group, Szentágothai Research Centre, University of Pécs and Institute of Biology, Faculty of Sciences, University of Pécs

Gábor Kemenesi ${ }^{18}$, Szentágothai Research Centre, University of Pécs, Pécs, Hungary Kornélia Kurucz ${ }^{19}$, Szentágothai Research Centre and Institute of Biology, Faculty of Sciences, University of Pécs, Pécs, Hungary

Virginia León-Règagnon ${ }^{20}$, Instituto de Biología, Universidad Nacional Autónoma de México, Mexico City, C.P. 04510

Hugo H. Mejía-Madrid21, Facultad de Ciencias, Departamento de Ecología y Recursos Naturales, Universidad Nacional Autónoma de México (UNAM), Ciudad Universitaria, Mexico City 04510 Mexico

Orsolya Molnár22, Institute of Evolution, Centre for Ecological Research, Karolina ut 29, Budapest, Hungary, $\mathrm{H}-1113$

Richard A. Nisbett ${ }^{23}$, Infectious Diseases of Poverty Field Epidemiology Unit, UL-PIRE Africa, Monrovia, Liberia

Wolfgang Preiser ${ }^{24}$, Professor and Head: Division of Medical Virology, Department of Pathology, Faculty of Medicine and Health Sciences, Stellenbosch University and NHLS Tygerberg

Francie van Zijl Rylaan Drive ${ }^{25}$, Tygerberg, Cape Town, South Africa

Michael Stuart ${ }^{26}$, Professor Emeritus, Department of Biology, University of North Carolina at Asheville, Asheville NC

Eors Szathmary ${ }^{27}$, Director General, Centre for Ecological Research, Karolina str 29, Budapest, Hungary, $\mathrm{H}-1113$, and Hungarian Academy of Sciences

Valeria Trivellone ${ }^{28}$, Illinois Natural History Survey, Prairie Research Institute, University of Illinois, Champaign, Illinois 61820 USA 


\section{Introduction}

On 30 January 2020 the World Health Organization (WHO) declared a Global Health Emergency of international concern attendant to the emergence and spread of SARS-CoV-2, nearly two months after the first reported emergence of human cases in Wuhan, China. In the subsequent two months, global, national and local health personnel and infrastructures have been overwhelmed, leading to suffering and death for infected people, and the threat of socio-economic instability and potential collapse for humanity as a whole. This shows that our current and traditional mode of coping, anchored in responses after the fact, is not capable of dealing with the crisis of emerging infectious disease. Given all of our technological expertise, why is there an emerging disease crisis, and why are we losing the battle to contain and diminish emerging diseases?

Part of the reason is that the prevailing paradigm explaining the biology of pathogenhost associations (coevolution, evolutionary arms races) has assumed that pathogens must evolve new capacities - special mutations - in order to colonize new hosts and produce emergent disease (e.g. Parrish and Kawaoka, 2005). In this erroneous but broadly prevalent view, the evolution of new capacities creates new opportunities for pathogens. Further, given that mutations are both rare and undirected, the highly specialized nature of pathogenhost relationships should produce an evolutionary firewall limiting dissemination; by those definitions, emergences should be rare (for a historical review see Brooks et al., 2019). Pathogens, however, have become far better at finding us than our traditional understanding predicts. We face considerable risk space for pathogens and disease that directly threaten us, our crops and livestock - through expanding interfaces bringing pathogens and hosts into increasing proximity, exacerbated by environmental disruption and urban density, fueled by globalized trade and travel. We need a new paradigm that explains what we are seeing.

\section{The Stockholm Paradigm}

The Stockholm Paradigm suggests that the capacity for pathogens to be associated with a given host is related to the occurrence of specific traits possessed by the host that represent required resources for the pathogen. The capacity for a pathogen to be associated with more than one host is related to how phylogenetically widespread those same resources are. How can the same capacity both restrict the range of hosts and create the possibility for changing hosts that characterizes emerging disease? The Stockholm Paradigm reverses the traditional perspective: most colonizations of new hosts are the result of pathogens taking advantage of new opportunities using pre-existing capacities; they do not represent the evolution of new, or special capacities, of pathogens.

In the case of SARS-CoV-2, specific receptors necessary for the virus to live successfully in bats are also broadly distributed among diverse mammalian groups (Parrish and Kawaoka 2005; Parrish et al., 2008; Hoffmann et al. 2020), making them all potential hosts. Any break-down in ecological isolation changes the dynamics for exposure because the array of hosts used in a particular environment (realized host range) is always a subset of all of the hosts that could potentially be infected (fundamental host range) (Agosta et al., 2010). The route from an original host to one we care about can be circuitous. When a pathogen has the opportunity to be exposed to an array of novel hosts, and becomes established in at least one, pathogen variants already present at low levels in the original host may find the new host more to their liking, in which case they will proliferate rapidly, and in turn produce additional variants that are capable of colonizing yet more hosts. This is called the steppingstone dynamic of host switching (Braga et al., 2014; Araujo et al., 2015; Brooks et al., 2019). 
SARS CoV-2 is a member of a large group of viral pathogens, the Coronaviruses, that occur normally in bats; but some rare variants are capable of inhabiting non-human hosts beyond Chiroptera (Kemenesi et al., 2014; Calderon et al., 2016; Joffrin et al., 2018; Zhou et al. 2020). Although the vast majority may not be transmitted directly to humans, numerous stepping-stone pathways can facilitate exposure and transmission (Araujo et al., 2015). Densely populated open-air markets where many exotic wildlife species are consumed as food can facilitate pathogen emergence. The original emergence of SARS-CoV in 2003 and SARS-CoV-2 in 2019 appear to have been driven by stepping-stone switches.

The Stockholm Paradigm suggests that the risk space for emerging diseases is immense. Our planet is a minefield of pathogens and potential emerging disease accidents waiting to happen (Brooks et al., 2019). Globalized trade and travel combined with climate change increase the chances of pathogen exposure across susceptible populations that have been historically isolated and naive to infections. Before the WHO announcement, emerging infectious diseases in human, crops, livestock and all wildlife upon which human depend socio-economically were costing the world more than $\$ 1$ trillion each year in treatment costs and production losses. To put that into context, only 15 countries have a GDP that large. SARS-CoV-2 is a rogue wave in an implacably rising tide.

The Stockholm Paradigm makes us realize that the emerging infectious disease crisis is associated with fundamental changes in the opportunity space for pathogens that facilitates mixing of pathogens, reservoirs and a broader array of potential hosts, rather than changes in the pathogens themselves. Three major elements of that altered space are: (1) Global climate change. Climate change catalyzes movement in many species, establishing new interfaces between humans and their domesticated species and an array of wildlife. Incursions into new habitats, or behaviors such as consumption of wildlife species, drive break-downs in the ecological isolation that previously confined pathogens to one or a few hosts; (2) Globalized trade and travel. Whether for curiosity, commerce or conflict, we move ourselves and associated species throughout the world to unprecedented degrees at unprecedented rates, increasing the diversity and frequency of pathogen encounters; and (3) Increasing urbanization. More than $50 \%$ of humanity now resides in cities, and the proportion is growing annually. Those who live in cities enjoy many advantages of technology and feel highly insulated from the impacts of many climate-related phenomena, including emerging diseases. And yet, cities - no matter how rich and powerful they seem - may be particularly vulnerable to disease. There are five reasons for this: (1) cities are warm - often $3 \mathrm{C}$ above the temperatures of peri-urban areas. This makes them ideal incubators for many pathogens and their vectors; (2) cities are totally dependent on a constant flow of materials, including water and food, from outside sources, increasing the chances for pathogen introduction; (3) cities support a large number of synurbic species of that are themselves vectors for diseases affecting both people and their pets (Földvári et al., 2011, 2014; Kurucz et al., 2018a,b). Urban green spaces add suitable habitat for pathogens and reservoir hosts and vectors to thrive in close proximity to humans. This threatens to offset the benefits of having urban green spaces; (4) cities represent high-density concentrations of people, meaning that in any disease outbreak the chances of exposure are elevated in proportion to the number of people living in the city. Furthermore, urban inhabitants have virtually no kinship affiliations with each other, so they are less likely to know who to trust and thus less likely to cooperate with each other; (5) cities exhibit extreme division of labor and extreme inter-dependency, meaning that there is little redundancy in the workforce to ensure continuity of services in case of disease outbreak; and (6) the inhabitants of every city include at least one sub-group who are poorlyeducated, poorly-nourished, and poorly-paid, working in jobs that bring physical exhaustion 
and exposure to stressful, often unsanitary, conditions. Such people are generally invisible to the public health and social services networks of the cities in which they live, and they cannot afford to stay at home when they are ill. They represent the urban Achilles Heel for disease introductions. And once a disease is established inside a city, it will not stay confined to the poor and invisible.

Paradoxically, the Stockholm Paradigm not only offers us a chilling view of the immensity of risk space for emerging diseases, it also gives us hope that we can do something truly proactive to mitigate the conditions leading to dissemination and emergence. The specialized capacities that allow disease-causing organisms to persist in one place give us insights into how they will behave in novel conditions. We can anticipate the arrival of disease-causing organisms and their behavior once they have become established; and that means we can mitigate their impacts on society. In order to do this, however, we must "find them before they find us."

\section{The DAMA Protocol}

The DAMA (Document-Assess-Monitor-Act) Protocol is the policy extension of the Stockholm Paradigm, providing a foundation for proactive activities to directly address potential and realized infectious diseases (Brooks et al., 2014; Hoberg and Brooks, 2015; Brooks et al., 2019). DAMA is now a global policy imperative. It helps provide a preventive dimension to traditional crisis-response modes of coping with infectious disease. Adopting and developing DAMA can extend human and material resources devoted to coping with the wave of emerging diseases that is only beginning and can buy time for development of new vaccines, medications, and control measures. Its focus is anticipating emerging diseases on the basis of knowing what pathogens are present in the environment before these become a problem, and then taking steps to diminish the risk space and to mitigate the impact of their emergence. Many countries recognize the magnitude of the EID crisis, but none have implemented a proactive national program taking substantial and effective actions to mitigate its impact on society. While the threats are global, preparedness has to be achieved locally. DAMA integrates activities ranging from the local "boots on the ground" contributions of citizen scientists led by field biologists to the most sophisticated technologies of archival repositories, bioinformatics, molecular biology and satellite surveillance.

Documenting the existence of disease-causing organisms actually or potentially residing in each country is fundamental. You cannot cope with disease-causing organisms unless you know who they are, where they occur, the pathways through which they circulate, and who they are likely to infect. Most, if not all, disease-causing organisms in humans, crops, and livestock reside in at least one host that is not diseased. Such reservoir hosts are often known or suspected, allowing us to focus on a manageable subset of all the species of plants and animals living in a given country. As well, those reservoirs most often live in habitats adjacent to places where humans, their crops and their livestock live. Disease transmission occurs in the interface between reservoir hosts in their preferred habitats and humans and economically important plants and animals in their habitats. And finally, the means by which disease-causing agents are transmitted from host to host are highly specific for each disease-causing organism - some are transmitted in food, some in water, some by contact between infected hosts or surfaces that have been in contact with infected hosts. Many are transmitted by other species, called vectors, such as mosquitoes and ticks. Datagathering of such a magnitude requires the cooperation of people living in the areas most at risk, especially the elders, who represent an immense storehouse of local knowledge, and the youth, who need to have that knowledge passed to them. Involved scientists need to be 
willing to expend significant time and energy talking with people at the grassroots, obtaining their trust and cooperation. Discovery leads to effective action only in the context of archival repositories.

Assessing the relative importance of pathogens encountered in documentation activities is crucial. We do not have enough time to examine everything we find in detail, so we must have a means of deciding where to focus. By applying what we know about the evolutionary history (phylogeny) of each species we find, we can quickly ask if the species or its relatives are known to cause disease in another place - this is phylogenetic triage. Species that fall into those categories are targeted for special attention. All others are archived for future reference if new information implicating them with disease becomes available (Dunnum et al. 2017).

Monitoring pathogens designated as significant in assessment leads to actions to mitigate their impact or making their arrivals less certain. Thus, within a selected habitat interface we can look to elements in common between reservoir hosts and those of interest to us to focus our search even more. We are looking for change - in geographic distribution, in host range, in transmission dynamics, in geographic variation, in what is there, and early signs of arrival of anticipated pathogens. The grandparent-grandchild coalition becomes vitally important at this level. Many cell phone-based apps have been developed - and more are coming - for rapid reporting of information from the grassroots to the centers for scientific assessment. Just as the grandparents have accumulated essential information about the local surroundings, the grandchildren are at home in the world of effective use of electronic communications.

Actions stemming from monitoring pathogens of special interest must effective and must be taken as rapidly as possible when necessary. Information about risks must be transmitted to the grassroots at the same time representatives of relevant governmental and NGO agencies are engaged. Every responsible organization must be committed to rapid and effective action based on the evidence at hand.

Information gathered by scientists has too seldom been converted into concrete action. A coronavirus with considerable genetic similarity to SARS-CoV-2 was discovered in bats from a cave in Yunnan Province, China more than 15 years ago (Poon et al., 2005; Li et al., 2005; Cyranoski, 2017) and the potential for such viruses to cause disease in humans was assessed shortly thereafter (Cheng et al., 2007). A pathogen in waiting was thus largely forgotten. The science that revealed this component of diversity was not converted to vital information. The metaphorical dots linking science to policy and action were never connected. The chance to anticipate and mitigate was not realized.

The EID crisis is thus a "health" issue in only a superficial sense. It's more fundamentally an evolutionary, ecological and biodiversity issue, a predictable consequence of separated species brought into close contact as a result of new opportunities provided by global climate change and human activities. Human population growth and commercial activity accelerates the rate of species introductions and establishment of suitable interfaces for new hosts be occupied, so outbreaks occur more frequently and over a wider geographic range than ever before. We have crossed tipping points from a large and slow world to one that is increasingly smaller and faster. Science has advanced rapidly, but not as rapidly as human population, slowing the effective application of new scientific information. The emergence of a powerful and highly risk-averse management class directing lines of research and mediating the exchange of scientific information, often in denial about the outcomes recognized by science, further exacerbates the problem. 


\section{A Sense of Urgency and Long-Term Commitment}

Given the state of the planet and our influence on it, it is not only a possibility that there will be a continuing parade of emerging and persisting pathogens, it is a certainty. A proactive response is our only pathway to effective mitigation, limiting the unsustainable costs of the emerging disease crisis. The time to initiate DAMA programs is now, even as the current pandemic burns to a hidden and cryptic pause, becoming another element of pathogen pollution with which health providers will have to deal in the future. We have an opportunity and a responsibility to push for an ethos of prevention and to move swiftly away from our business as usual reactive mode of crisis response.

Make no mistake - we are in a war for the survival of technological humanity and we are losing that war. There is always hope, but each day of inaction increases the odds of catastrophic outcomes. We need to be proactive, not reactive. To date, the human, agricultural, veterinary, and wildlife disease communities have been in reactive mode. This is due, in large part, to an ancient philosophical dictum that health providers should "Do No Harm." In times of global climate change, this dictum becomes a mandate to do nothing until a crisis manifests itself, at which point it is too late to enact cost-effective policies.

The challenge of adapting to a world in which global climate change is a real and long-lasting phenomenon requires long-term commitment to changes in education, human capacity and infrastructure. These actions can no longer be short-term interventions, but rather will require permanent societal investments in new forms of education, scientific research, and clinical practices. These efforts will be is costly, but only a miniscule fraction of the dramatic impacts in human suffering and economic collapse that are driven by emergent disease. Not implementing them dooms us to continue current economically unsustainable practices.

Sadly, there is nothing any country can do that will not cost more money. But we are beyond the point at which economizing in the short term is possible. Implementing the DAMA protocol nationally and participating in DAMA activities internationally will not be cheap, especially in the area of revitalizing and augmenting archival capacities and associated informatics services (Schindel and Cook 2018, McLean et al. 2019). Not implementing the DAMA protocol, however, will be catastrophic. The costs of pathogen discovery and information development are negligible when weighed against the cost of the economic and societal impacts of addressing emergent disease after the fact.

We cannot stop pathogens from coming to us as a result of opportunities provided by the linked phenomena of climate change, globalization and urbanization. We can, however, be prepared for them when they arrive. We have the capacity not to be continually surprised by emerging pathogens and their socio-economic impact. 


\section{References}

Agosta S, Janz N, Brooks DR (2010) How generalists can be specialists: resolving the "parasite paradox" and implications for emerging disease. Zoologia 27:151-162

Araujo SBL, Braga MP, Brooks DR, Agosta S, Hoberg EP, von Hathental F, Boeger WA. (2015)

Understanding host-switching by ecological fitting. PLoS ONE 10(10): e0139225. doi:10.1371/ journal. pone.0139225.

Braga MP, Razzolini E, Boeger WA (2014) Drivers of parasite sharing among Neotropical freshwater fishes. J. An. Ecol. 84:487-97

Brooks DR, Hoberg EP, Boeger WA (2019) The Stockholm Paradigm: Climate Change and Emerging Disease. University of Chicago Press, Chicago. 423 p.

Brooks, DR, Hoberg EP, Gardner SL, Boeger W, Galbreath KE, Herczeg D, Mejía-Madrid $\mathrm{HH}$,

Racz E, Tsogtsaikhan Dursahinhan A (2014) Finding them before they find us: Informatics, parasites and environments in accelerating climate change. Comparative Parasitology 81:155-164

Calderon, A., Guzman, C., Salazar-Bravo, J., Figueiredo, L.T., Mattar, S. and Arrieta, G. (2016)

Viral zoonoses that fly with bats: a review. Manter: Journal of Parasite Biodiversity. 6:1-13.

Cheng VCC, Lau SKP, Woo PCY, Yuen KY (2019) Severe Acute Respiratory Syndrome coronavirus as an agent of emerging and reemerging infection. Clin. Microbiol. Rev. 20: 660-694

Cyranoski D (2017) SARS outbreak linked to Chinese bat cave. Nature 522:15-16

Dunnum JL, Yanagihara R, Johnson KM, Armien B, Batsaikhan N, Morgan L, Cook JA (2017) Biospecimen repositories and integrated databases as critical infrastructure for pathogen discovery and pathobiology research. PloS Negl. Trop. Dis. 11: e0005133. doi: 10.1371/journal.pntd.0005133

Fan Y, Zhao K, Shi Z L, Zhou P (2019) Bat Coronaviruses in China. Viruses 11: doi:10.3390/ v11030210

Földvári, G, Jahfari S, Rigó K, Jablonszky M, Szekeres S, Majoros G, Tóth M, Molnár V, Colpan EC, Sprong H (2014) Candidatus neoehrlichia mikurensis and Anaplasma phagocytophilum in Urban Hedgehogs. Emerging Infectious Diseases 20: 496-97 doi:10.3201/eid2003.130708.

Földvári, G, Rigó K, Jablonszky M, Biró N, Majoros G, Molnár V, Tóth M (2011) Ticks and the city: ectoparasites of the Northern White-Breasted Hedgehog (Erinaceus roumanicus) in an Urban Park. Ticks and Tick-Borne Diseases 2:231-34 doi:10.1016/j.ttbdis.2011.09.001. Hoberg EP, Brooks DR (2015) Evolution in action: climate change, biodiversity dynamics and emerging infectious disease. Phil. Trans. Royal Soc. B 370: 20130553.

Hoffmann M, Kleine-Weber H, Schroeder S, Krüger N, Herrler T, Erichsen S, Schiergens TS, Herrler G, Wu N-H, Nitsche A, Müller MA, Drosten C, Pöhlmann S (2020) SARS-CoV-2 Cell Entry Depends on ACE2 and TMPRSS2 and Is Blocked by a Clinically Proven Protease Inhibitor. Cell https://doi.org/10.1016/j.cell.2020.02.052

Joffrin L, Dietrich M, Mavingui P, Lebarbenchon C (2018) Bat pathogens hit the road: but which one? PLoS Pathogens. August 9, 2018 https://doi.org/10.1371/journal.ppat.1007134

Kemenesi G, Dallos B, Görfö T, Boldogh S, Estók P, Kurucz K, Kutas A, Földes F, Oldal M, Németh V, Martella V, Bányai K,7, Jakab F (2014) Molecular survey of RNA viruses in Hungarian Bats: Discovering Novel Astroviruses, Coronaviruses, and Caliciviruses. Vector-borne and zoonotic diseases 14: 846-855 
Kurucz K, Madai M, Bali D, Hederics D, Horváth G, Kemenesi G, Jakab F (2018a) Parallel Survey of Two Widespread Renal Syndrome-Causing Zoonoses:Leptospira spp. and Hantavirus in Urban Environment, Hungary. Vector-borne and zoonotic diseases 18: 200-205

Kurucz K, Hederics D, Bali D, Kemenesi G, Horváth G, Jakab F. (2018b) Hepatitis E virus incommon voles (Microtus arvalis) from an urban environment, Hungary: discovery of a Cricetidae-specific genotype of Orthohepevirus C. Zoonoses and Public Health 2018:1-5. https://doi.org/10.1111/zph.12543

Li W, Shi Z, Yu M, Ren W, Smith C, Epstein JH, Wang H, Crameri G, Hu Z, Zhang H, Zhang J, McEachern J, Field H, Daszak P, Eaton BT, Zhang S, Wang LF (2005) Bats are natural reservoirs of SARS-like coronaviruses. Science 310: 676-679

McLean BS, Cook JA, Hoberg EP, Durden LA, Guralnick RP (2019) The next chapter of human-plague science. Proc. Nat. Acad. Sci. USA 116:14411-14412

Parrish CR, Kawaoka Y. (2005) The origins of new pandemic viruses: the acquisition of new host ranges by canine parvovirus and influenza A viruses. Annu. Rev. Microbiol. 59: 553-586

Parrish CR, Holmes EC, Morens DM, Park E-C, Burke DS, Calisher CH, Laughlin CA, Saif LJ, Daszak P (2008) Cross-species virus transmission and the emergence of new epidemic diseases. Microbiol. Molec. Biol. Rev. Sept. 2008: 457-470.

Poon LLM, Chu DKW, Chan KH, Wong OK, Ellis TM, Leung YHC, Lau SKP, Woo PCY, Suen KY, Yuen KY, Guan Y, Peiris JSM (2005). Identification of a Novel Coronavirus in Bats. J. Virol. 79:2001-2009

Schindel DE, Cook JA (2018) The next generation of natural history collections. PLoS Biology 16: e2006125

Zhou P, Yang X, Wang X, Hu B, Zhang L, Zhang W, Si H, Zhu Y, Li B, Huang C, Chen H, Chen J, Luo Y, Guo H, Jiang R, Liu M, Chen Y, Shen X, Wang X, Zheng X, Zhao K, Chen Q, Deng F, Lin-Lin Liu L, Yan B, Zhan F, Wang Y, Xiao G, Shi Z (2020) A pneumonia outbreak associated with a new coronavirus of probable bat origin. Nature 579:270-273 https://doi.org/10.1038/s41586-020-2012-7 
